\title{
A pilot study of regenerative therapy using controlled release of recombinant human fibroblast growth factor for patients with pre-collapse osteonecrosis of the femoral head
}

\author{
Yutaka Kuroda $^{1} \cdot$ Ryuta Asada $^{2} \cdot$ Kazutaka So $^{1} \cdot$ Atsushi Yonezawa $^{3}$. \\ Manabu Nankaku ${ }^{4} \cdot$ Kumi Mukai $^{5}$ - Toshiko Ito-Ihara ${ }^{5} \cdot$ Harue Tada $^{6}$. \\ Michio Yamamoto ${ }^{6} \cdot$ Toshinori Murayama $^{5} \cdot$ Satoshi Morita $^{6}$ - Yasuhiko Tabata ${ }^{8}$. \\ Masayuki Yokode $^{5} \cdot$ Akira Shimizu $^{7} \cdot$ Shuichi Matsuda ${ }^{1} \cdot$ Haruhiko Akiyama $^{9}$
}

Received: 11 September 2015 / Accepted: 14 December 2015 /Published online: 29 December 2015

(C) The Author(s) 2015. This article is published with open access at Springerlink.com

\begin{abstract}
Purpose We evaluated the safety and clinical outcomes of a single local administration of gelatin hydrogel impregnated with recombinant human fibroblast growth factor (rhFGF)-2 for the treatment of the precollapse stage of osteonecrosis of the femoral head (ONFH).

Methods Patients with ONFH (precollapse stage $\leq 2$ ) received a single local administration of $800 \mu \mathrm{g}$ of rhFGF-2impregnated gelatin hydrogel and were followed up for one year. The surgery was performed using a minimally invasive technique involving a $1-\mathrm{cm}$ skin incision, and walking was allowed from day one postoperatively. The primary outcomes included occurrence of adverse events and complications. The secondary outcomes included changes in the Harris hip scores, visual analog scale for pain scores, University of California, Los Angeles (UCLA) activity scores, and radiological images. Results We included ten patients, of which five experienced 14 adverse events, including one complication from spinal anesthesia. However, patients completely recovered from all adverse events. The mean clinical scores significantly
\end{abstract}

Yutaka Kuroda

ykuromd@kuhp.kyoto-u.ac.jp

1 Department of Orthopaedic Surgery, Graduate School of Medicine, Kyoto University, Shogoin, Kawahara-cho 54,

Sakyo-ku, Kyoto 606-8507, Japan

2 Clinical Research Center, Gifu University Hospital, Gifu, Japan

3 Department of Clinical Pharmacology and Therapeutics, Kyoto University Hospital, Kyoto, Japan

4 Rehabilitation Unit, Kyoto University Hospital, Kyoto, Japan improved by one year postoperatively compared with the pre-operative scores (before vs. after: visual analog score for pain, 21.2 vs. $5.3 \mathrm{~mm}$; UCLA activity score, 5.5 vs. 6.6; Harris hip score, 81.0 vs. 96.9 points). There was only one case of femoral head collapse; however, this occurred in a hip with extensive necrosis. Stage progression and collapse did not occur in the other nine cases. Computed tomography confirmed bone regeneration in the femoral heads.

Conclusions Clinical application of rhFGF-2-impregnated gelatin hydrogel for patients with precollapse ONFH was feasible and safe.

Keywords Clinical trial $\cdot$ Femoral head $\cdot$ FGF $\cdot$ Growth factor · Osteonecrosis $\cdot$ Regenerative therapy
Abbreviations
$\mathrm{AE}$
Adverse event
CT Computed tomography

5 Department of Clinical Innovative Medicine, Institute for Advancement of Clinical and Translational Research (iACT), Kyoto University Hospital, Kyoto, Japan

6 Department of Data Science, Institute for Advancement of Clinical and Translational Research (iACT), Kyoto University Hospital, Kyoto, Japan

7 Department of Experimental Therapeutics, Institute for Advancement of Clinical and Translational Research (iACT), Kyoto University Hospital, Kyoto, Japan

8 Department of Biomaterials, Field of Tissue Engineering, Institute for Frontier Medical Sciences, Kyoto University, Kyoto, Japan

9 Department of Orthopaedic Surgery, Gifu University, Gifu, Japan 


$\begin{array}{ll}\text { HHS } & \text { Harris hip score } \\ \text { JICHW } & \begin{array}{l}\text { Japanese Investigation Committee of Health } \\ \text { and Welfare }\end{array} \\ \text { MRI } & \text { Magnetic resonance imaging } \\ \text { ONFH } & \text { Osteonecrosis of the femoral head } \\ \text { rhFGF } & \text { Recombinant human fibroblast growth factor } \\ \text { THA } & \text { Total hip arthroplasty } \\ \text { UCLA } & \text { University of California, Los Angeles } \\ \text { VAS } & \text { Visual analog scale }\end{array}$

\section{Introduction}

Osteonecrosis of the femoral head (ONFH) is a multifactorial disease that can cause femoral head collapse, pain, gait disorders, and secondary hip osteoarthritis. Several causative factors have been indicated, including hypercoagulation, bone marrow fat embolisms, elevated internal pressure in the femoral head, and vascular endothelial dysfunction; however, the cause has not yet been elucidated completely. ONFH is common among young people in their $30 \mathrm{~s}$ and $40 \mathrm{~s}$, and it bilaterally occurs in approximately $50 \%$ cases.

Magnetic resonance imaging (MRI) can be useful for early diagnosis; however, initial-stage symptoms are usually mild, with pain intensifying after femoral head collapse. In the clinic, although patients are diagnosed, 70-80\% of untreated patients experience femoral head collapse and have to undergo total hip arthroplasty (THA) [1]. Thus, research over the past decade has focused on identifying a minimally invasive regenerative therapy that can help prevent femoral head collapse [2, 3].

Therapies using cells, proteins, and other bone growthpromoting substances have been proposed, and various types of autologous marrow cell therapy have been used [4-6]. Furthermore, non-cellular therapeutic strategies that use growth factors have also been proposed [2, 3, 7, 8]. In an animal study using adult rabbits, we reported that a single local administration of gelatin hydrogel impregnated with recombinant human fibroblast growth factor (rhFGF)-2 not only promoted the regeneration of the necrotic bone but also suppressed the progression of ONFH [9].

The aim of this preliminary clinical study was to evaluate the safety and efficacy of a single local administration of rhFGF-2-impregnated gelatin hydrogel for the treatment of the pre-collapse stage of ONFH in humans. Here we provide a summary report of the trial and the short-term results.

\section{Materials and methods}

\section{Ethics}

The treatment protocol was approved by the Ethics Committee of Kyoto University Graduate School and Faculty of
Medicine. Before enrollment, written informed consent was obtained from each participant included in the study. The study was registered with the public Japanese clinical trials registry, the University Hospital Medical Information Network Clinical Trials Registry (UMIN000009250).

\section{Study design}

We designed this phase II, prospective, open-label clinical trial to evaluate the safety and clinical outcomes of a single local administration of rhFGF-2-impregnated gelatin hydrogel as a treatment for the precollapse stage of ONFH. The study was conducted at a single institution over 12 months, with patients enrolled and treated between March 1, 2013, and March 27, 2013, with follow-up ending on June 19, 2014.

\section{Patients}

The eligibility criteria were age between 20 and 80 years and presence of ONFH at precollapse stage 1 or 2 according to the classification system for ONFH staging developed by the Japanese Investigation Committee of Health and Welfare (JICHW) [10]. Factors underlying ONFH were determined as associated with steroid use, associated with alcohol intake, or idiopathic.

\section{Surgical technique and rehabilitation}

Patients were placed in the supine position on a fracture operation table with a C-arm image intensifier. A 1-cm skin incision was created over the lateral aspect of the femur near the level of the lesser trochanter, and $800 \mu \mathrm{g}$ of rhFGF-2-impregnated gelatin hydrogel was embedded percutaneously. In brief, under fluoroscopic control, a 2.4-mm Kirschner guide wire was inserted in the direction of the ONFH lesion. The first drilling was carefully directed to the center of the ONFH region using a $\mathrm{C}$-arm image based on preoperative planning. With a softtissue protector in place, core decompression was performed via the guide wire with a $4.8-\mathrm{mm}$ diameter trephine. A $3.5-\mathrm{mm}$ cylinder was inserted into the core decompression site, and the hydrogel was inserted in the ONFH region using a special pusher (Iso Medical Systems, Tokyo, Japan).

Weight bearing was prohibited on the day of surgery; however, walking was encouraged under the guidance of physical therapists on day one post-operatively. Before treatment, the physical therapist assessed walking capability, including passive hip motion, in all participants.

\section{Preparation of rhFGF-2-impregnated gelatin hydrogel}

Recombinant hFGF-2 was provided by Kaken Pharmaceutical Co. (Tokyo, Japan). For controlled-release, biodegradable gelatin hydrogel was prepared using glutaraldehyde cross-linking 
of acidic gelatin purified from natural bovine bone, as reported previously $[11,12]$.

\section{Safety assessment}

Safety was monitored by the Department of Clinical Trial Design and Management Translational Center according to the occurrence and severity of adverse events (AEs). AEs were defined as any sign of worsening of the patients' condition after treatment, and they were classified as either serious or non-serious. AEs requiring hospitalization were classified as serious and were reported and assessed by an external data monitoring committee, with the severity classified as mild, moderate, or severe. Causal relationships between the clinical trial and resolution of each $\mathrm{AE}$ were judged and evaluated.

\section{Clinical assessment}

All participants underwent preoperative clinical assessment, and they were prospectively followed-up clinically and radiographically. Patients were evaluated at day one, three, six, and 12 months post-operatively. The primary endpoint was safety assessment, and the secondary endpoints were changes in the clinical scores, radiographic stage of ONFH, and bone regeneration as evaluated by radiography, computed tomography (CT), and MRI.

The following clinical score tools were used at baseline (pre-operative) and at three, six, and 12 months (post-operatively). Patients were evaluated using the visual analog scale (VAS) for pain, the University of California, Los Angeles (UCLA) activity score [13], and the Harris hip score (HHS) [14]. VAS for pain is a self-assessment scale for patients, which ranges from $0 \mathrm{~mm}$ (no pain) to $100 \mathrm{~mm}$ (maximum pain). The UCLA activity score uses a simple scale ranging from 1 to 10 . A rating of 1 is defined as "no physical activity, dependent on others" and 10 is defined as "regular participation in impact sports." HHS ranges from 0 to 100.025 points, with higher scores indicating better outcomes, and has the following subcategories: pain (44 points), limp (11 points), support (11 points), distance walked (11 points), sitting (5 points), stairs (4 points), putting on shoes and socks (4 points), absence of deformity (4 points), and range of motion (5.025 points). HHS for range of motion was assessed by a physical therapist. For patients with bilateral hip involvement, both hips were examined.

\section{Radiographic assessment}

Serial anteroposterior and frog lateral radiographs of the affected hip were used to examine radiographic progression of the femoral head collapse in hips (before surgery to one year follow-up) according to each JICHW classification stage. The JICHW stage and type classifications were used to describe
ONFH and localization of the necrotic lesion [10]. During preoperative planning, the drilling route to the necrotic lesion was planned using navigation software (Orthomap 3D, Stryker, Kalamazoo, MI, USA; Fig. 1). This software was used to evaluate bone regeneration at the implanted area of the femoral head. The fraction of osteonecrotic volume to the whole femoral head was calculated using integrated data from CT and MRI scans. To clarify the location of bone regeneration in CT images, further radiological analysis (not included in the protocol of the clinical trial) was performed. The coronal CT images obtained pre-operatively were compared with those obtained at the one year post-operative follow-up after converting into a binary data format using Image $J$ software (U.S. National Institutes of Health, Bethesda, MD, USA). For evaluating binary format data, thresholds for each specimen were determined at the same condition used to clarify if and where the bone regenerated. The difference in the two binary format images was defined as the regenerated bone region, as described previously [9].

\section{Statistical analysis}

All statistical analyses were performed on an intention-to-treat basis. Safety and efficacy analyses were conducted on all patients who received treatment. The incidence of AEs and baseline characteristics were described for each patient. The changes over time in the VAS for pain scores, UCLA activity scores, and HHSs were summarized and investigated using a repeated-measures linear mixed-effect model containing terms for time with a compound symmetry covariance structure [15]. The effect of time was analyzed with a one-tailed $t$ test. Advances in the disease stage and the incidence of femoral head collapse were evaluated for each patient by radiographic evaluation. Statistical analyses were performed using SAS version 9.3 software (SAS Institute, Inc., Cary, NC, USA). All data are reported as mean \pm standard deviation. The linear mixed-effect model was used for statistical analyses, unless indicated otherwise. All $P$ values $<0.05$ were considered significant.

\section{Results}

\section{Demographics}

We included five men (five hips) and five women (five hips), with a mean age of 39.8 years (range: $29-53$ years) at the time of surgery (Table 1). Although eight patients had bilateral ONFH, three had already undergone THA on the contralateral side (cases 1,2, and 8). At the first medical examination, two patients with bilateral disease (cases 4 and 10) had already been scheduled for THA on the contralateral side. Eight patients were receiving treatment with corticosteroid therapy, 

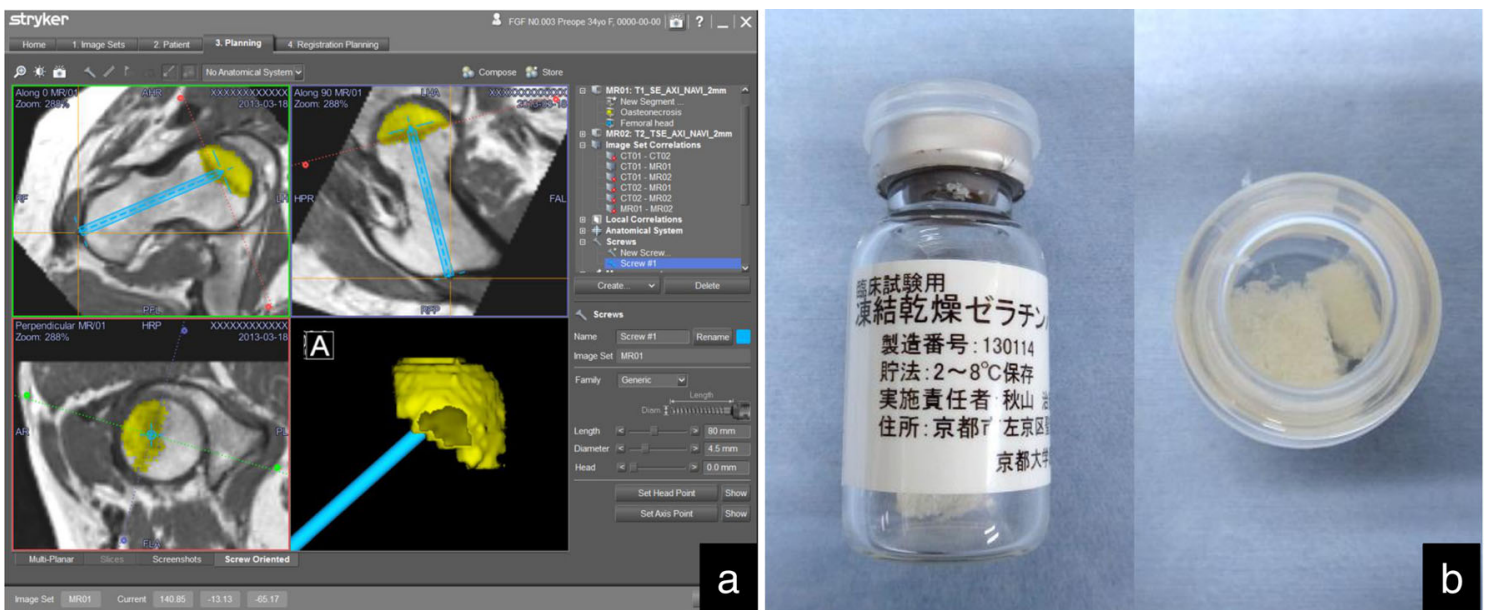
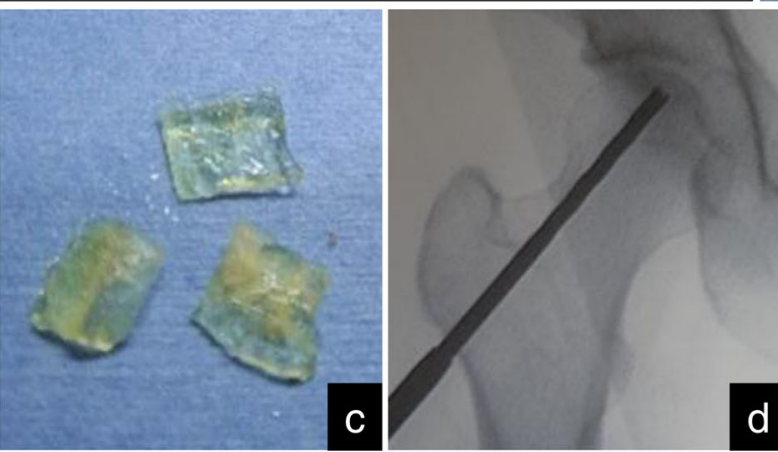

Fig. 1 Representative pre-operative planning and intra-operative photographs. a A typical screenshot of pre-operative planning is shown. The yellow area shows the area of osteonecrosis. The surgeon planned the route and drilling position (blue screw). The planning tool provided essential guidance for the first drilling, which was critical to the success of the procedure. The necrosis volume fraction of the femoral head was calculated by integrating data from magnetic resonance and computed tomography images. b Preparation of the recombinant human fibroblast

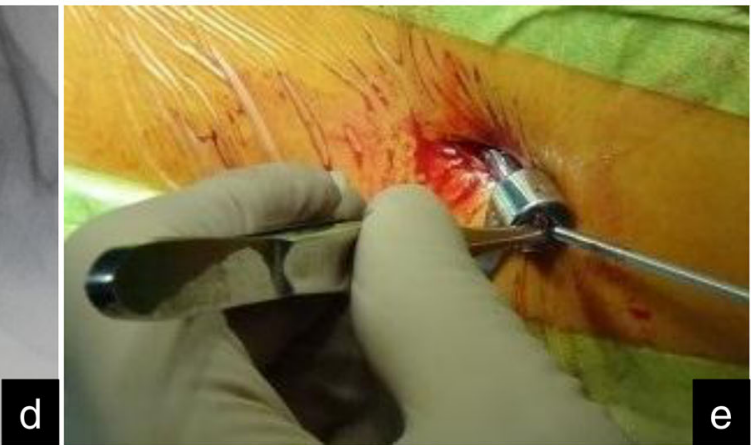

growth factor (rhFGF)-2-impregnated gelatin hydrogel. The gelatin hydrogel was bottled with a mandatory 30 minute impregnation time for the rhFGF-2 solution. c Representative pieces of the rhFGF-2impregnated gelatin hydrogel. d A representative intra-operative fluoroscopic image after drilling. e A representative intra-operative photograph showing administration of the gelatin hydrogel using a percutaneous technique

Table 1 Patient demographics and baseline characteristics

\begin{tabular}{|c|c|c|c|c|c|c|}
\hline Case & Age/sex & Background factors for ONFH & $\begin{array}{l}\text { Laterality of ONFH } \\
\text { (operated side) }\end{array}$ & Stage $\mathrm{R} / \mathrm{L}$ & Type R/L & $\begin{array}{l}\text { Necrosis volume } \\
\text { (percentage) }\end{array}$ \\
\hline 1 & $30 / F$ & Alcohol intake & Bilateral (R) & $2 / \mathrm{THA}$ & $\underline{\mathrm{A} /-}$ & $1.8 \mathrm{~cm}^{3}(5.1 \%)$ \\
\hline 2 & $53 / \mathrm{F}$ & Steroid use (asthma) & Bilateral (L) & $\mathrm{THA} / 2$ & $-\bar{B}$ & $3.0 \mathrm{~cm}^{3}(8.3 \%)$ \\
\hline 3 & $34 / F$ & Steroid use (V-K-H synd.) & Unilateral (R) & $\underline{1} /-$ & $\underline{\mathrm{C} 1} /-$ & $10.7 \mathrm{~cm}^{3}(25.7 \%)$ \\
\hline 4 & $35 / \mathrm{M}$ & Alcohol intake & Bilateral (R) & $2 / 3 \mathrm{~A}$ & $\overline{\mathrm{C} 2} / \mathrm{C} 2$ & $39.7 \mathrm{~cm}^{3}(72.3 \%)$ \\
\hline 5 & $51 / \mathrm{M}$ & Steroid use (cutaneous pruritus) & Bilateral (R) & $\underline{2} / 3 \mathrm{~A}$ & $\mathrm{C} 2 / \mathrm{C} 2$ & $13.3 \mathrm{~cm}^{3}(23.5 \%)$ \\
\hline 6 & $41 / \mathrm{M}$ & Steroid use (nephrotic syndrome) & Bilateral (R) & $2 / 3 \mathrm{~A}$ & $\overline{\mathrm{C} 2} / \mathrm{C} 1$ & $23.6 \mathrm{~cm}^{3}(41.5 \%)$ \\
\hline 7 & $29 / \mathrm{M}$ & Steroid use (AGA) & Bilateral (R) & $2 / 3 \mathrm{~A}$ & $\overline{\mathrm{C} 2} / \mathrm{C} 1$ & $12.0 \mathrm{~cm}^{3}(20.4 \%)$ \\
\hline 8 & $47 / \mathrm{M}$ & Steroid use (nephrotic syndrome) & Bilateral (R) & $\underline{2} / \mathrm{THA}$ & $\mathrm{C} 2 /-$ & $11.3 \mathrm{~cm}^{3}(23.1 \%)$ \\
\hline 9 & $32 / \mathrm{F}$ & Steroid use (SLE) & Unilateral (L) & $-/ 2$ & $-/ \underline{\mathrm{C} 2}$ & $9.0 \mathrm{~cm}^{3}(26.2 \%)$ \\
\hline \multirow[t]{2}{*}{10} & $46 / F$ & Steroid use (SLE) & Bilateral (L) & $4 / \underline{2}$ & $\mathrm{C} 2 / \underline{\mathrm{C} 2}$ & $9.8 \mathrm{~cm}^{3}(19.8 \%)$ \\
\hline & Mean: 39.8/M:F $=5: 5$ & $\begin{array}{l}\text { Steroid use: } 8 \\
\text { Alcohol intake: } 2 \\
\text { Idiopathic: } 0\end{array}$ & $\begin{array}{l}\text { Bilateral: } 8 \\
\text { Unilateral: } 2 \\
(\mathrm{R}: \mathrm{L}=7: 3)\end{array}$ & $\begin{array}{l}\text { Stage } 1: 1 \\
\text { Stage } 2: 9\end{array}$ & $\begin{array}{l}\text { Type A: } 1 \\
\text { Type B: } 1 \\
\text { Type C1: } 1 \\
\text { Type C2: } 7\end{array}$ & Mean: $23.8 \mathrm{~cm}^{3}(27.4 \%)$ \\
\hline
\end{tabular}

Abbreviations: M, male; F, female; ONFH, osteonecrosis of the femoral head; R, right; L, left; THA, total hip arthroplasty; V-K-H synd., VogtKoyanagi-Harada syndrome; AGA, allergic granulomatous angiitis; SLE, systemic lupus erythematosus. Underline indicates the operated side 
and two patients overused alcohol. Stage 1 and 2 disease was present in one and nine patients, respectively. One patient each had type $\mathrm{A}$, type $\mathrm{B}$, and type $\mathrm{C} 1$ disease, whereas seven patients had a type $\mathrm{C} 2$ lesion. Pre-operatively, the mean necrosis volume and necrosis volume fraction (percentage of total tissue) was $23.8 \mathrm{~cm}^{3}\left(1.8-39.7 \mathrm{~cm}^{3}\right)$ and $27.4 \%(5.1 \%-$ $72.3 \%)$, respectively.

\section{Safety}

We evaluated all patients over a one year follow-up period (Table 2). Fourteen AEs were experienced by five patients during the observation period. There were two serious AEs, classified to be of moderate severity (cases 4 and 10), in which the contralateral side was diagnosed as advancing to above stage 3 and ONFH. However, these were not associated with the clinical trial, and the patients had already been scheduled for THA. Regarding causal relationships, one patient (case 10) was judged to have developed headache due to spinal anesthesia. In all cases, the patients recovered from these AEs over the follow-up period. There were no complications related to the surgical technique.

\section{Clinical outcomes}

All procedures were performed under spinal anesthesia. The mean operation time was 18 minutes, the skin incision was $1 \mathrm{~cm}$, there was little bleeding, walking was encouraged from day one post-operatively, and the mean hospital stay was 6.2 days (range: 5-7 days). Embedding of the rhFGF-2impregnated gelatin hydrogel in the necrotic area was confirmed in all cases. The mean clinical scores improved significantly by one year post-operatively (VAS for pain, $5.3 \mathrm{~mm}$;
UCLA activity score, 6.6; HHS, 96.9 points) compared with pre-operative scores (VAS for pain, $21.2 \mathrm{~mm}$; UCLA activity score, 5.5; HHS, 81.0 points) (Table 3 ).

\section{Radiographic evaluation}

Although one patient (case 4) developed femoral head collapse $(10 \%)$ at the one year post-operative follow-up, this patient had the greatest necrosis volume fraction. Moreover, based on post-operative CT images obtained at day one postoperatively, femoral head collapse was considered to have progressed from an early collapse that had already been present at the time of surgery.

The remaining nine patients neither showed progression of pre-operative ONFH nor did they exhibit femoral head collapse during the follow-up period. Changes in the necrosis volume fraction and ONFH type were not observed in the necrotic areas by MRI; however, there was evidence of homogeneous low-signal areas in postoperative T1-weighted images of the femoral heads (Fig. 2). In radiographs and CT scans, bone regeneration was observed in the necrotic regions in all cases. Additional radiographic assessment using binary format images showed regeneration of the trabecular bones and subchondral bone (Fig. 3).

\section{Discussion}

The minimally invasive treatment described in this study aimed to prevent femoral head collapse by direct local administration of rhFGF-2, which has both angiogenic and osteogenic actions. To the best of our knowledge, there have been

Table 2 Adverse events

\begin{tabular}{lllll}
\hline No. & Adverse events & Degree of seriousness & Level of severity & Outcome \\
\hline 2 & Muscle contusion & Non-serious & Moderate & Recovered \\
3 & Chronic gastritis & Non-serious & Mild & Recovered \\
3 & Panic disorder & Non-serious & Mild & Recovered \\
3 & Loss of hair & Non-serious & Mild & Recovered \\
3 & Cystitis & Non-serious & Mild & Recovered \\
4 & AST and ALT elevation & Non-serious & Mild & Recovered \\
4 & Pain on the opposite side & Non-serious & Mild & Recovered \\
4 & Pain on the affected side & Non-serious & Mild & Recovered \\
4 & Pain on the affected side & Non-serious & Mild & Recovered \\
4 & THA on the opposite side & Serious & Moderate & Recovered \\
7 & Cough & Non-serious & Mild & Recovered \\
10 & Headache & Non-serious & Mild & Recovered \\
10 & Herpes & Non-serious & Mild & Recovered \\
10 & THA on the opposite side & Serious & Moderate & Recovered \\
\hline
\end{tabular}

Abbreviations: No., patient number; THA, total hip arthroplasty; AST, aspartate aminotransferase; ALT, alanine transaminase. 
Table 3 Clinical outcomes

\begin{tabular}{clllll}
\hline Score (range) & Pre-operative & $\begin{array}{l}\text { Post-operative } \\
3 \text { months }\end{array}$ & $\begin{array}{l}\text { Post-operative } \\
6 \text { months }\end{array}$ & $\begin{array}{l}\text { Post-operative } \\
12 \text { months }\end{array}$ & P value \\
\hline VAS for pain & $21.2 \pm 24.9$ & $6.1 \pm 7.8$ & $5.3 \pm 5.5$ & $5.3 \pm 8.6$ & 0.002 \\
$(0-100)$ & $10.0(0-84)$ & $3.5(0-25)$ & $4.5(0-14)$ & $1.0(0-27)$ & \\
UCLA activity & $5.5 \pm 1.0$ & $6.1 \pm 0.7$ & $6.1 \pm 0.7$ & $6.6 \pm 0.5$ & $<0.001$ \\
$(1-10)$ & $6(4-7)$ & $6(5-7)$ & $6(5-7)$ & $7(6-7)$ & \\
HHS (0-100) & $81.0 \pm 18.1$ & $94.8 \pm 4.0$ & $94.5 \pm 7.9$ & $96.9 \pm 5.0$ & $<0.001$ \\
& $91.9(44.9-$ & $95.0(88.0-100.0)$ & $97.0(74.0-100.0)$ & $100.0(85.9-$ & \\
& $96.0)$ & & & $100.0)$ & \\
\hline
\end{tabular}

Data for each score are expressed as mean \pm standard deviation (the upper rows) and median range (the bottom rows). The $\mathrm{P}$ value was calculated for the effect of time in a repeated-measures linear mixed-effect model. Abbreviations: VAS, visual analog scale; UCLA, University of California, Los Angeles activity; HHS, Harris hip score

no previous reports regarding the administration of rhFGF-2impregnated gelatin hydrogel into the femoral head.

During the one year follow-up period, 14 AEs occurred in five patients, and all recovered without problems. There were no complications related to either the surgical technique or treatment. Moreover, all clinical scores related to pain, daily activity rating, and hip joint functions significantly improved post-operatively. Although one patient with stage $3 \mathrm{~A}$ disease at surgery (case 4) developed early femoral head collapse that was present on CT images at day one post-operatively, no other patients showed disease progression or femoral head collapse. Thus, local administration of rhFGF-2 was safe.

Therapies using cells [4-6], proteins, and other bone growth-promoting substances [2, 7, 8] have been proposed, and various cell therapies using autologous marrow cells are already being attempted. Non-cellular therapeutic strategies using growth factors have been proposed; however, there has been little progress with regard to the verification of animal experiments, primarily because of the absence of an animal model for femoral head-specific necrosis [9, 16] and
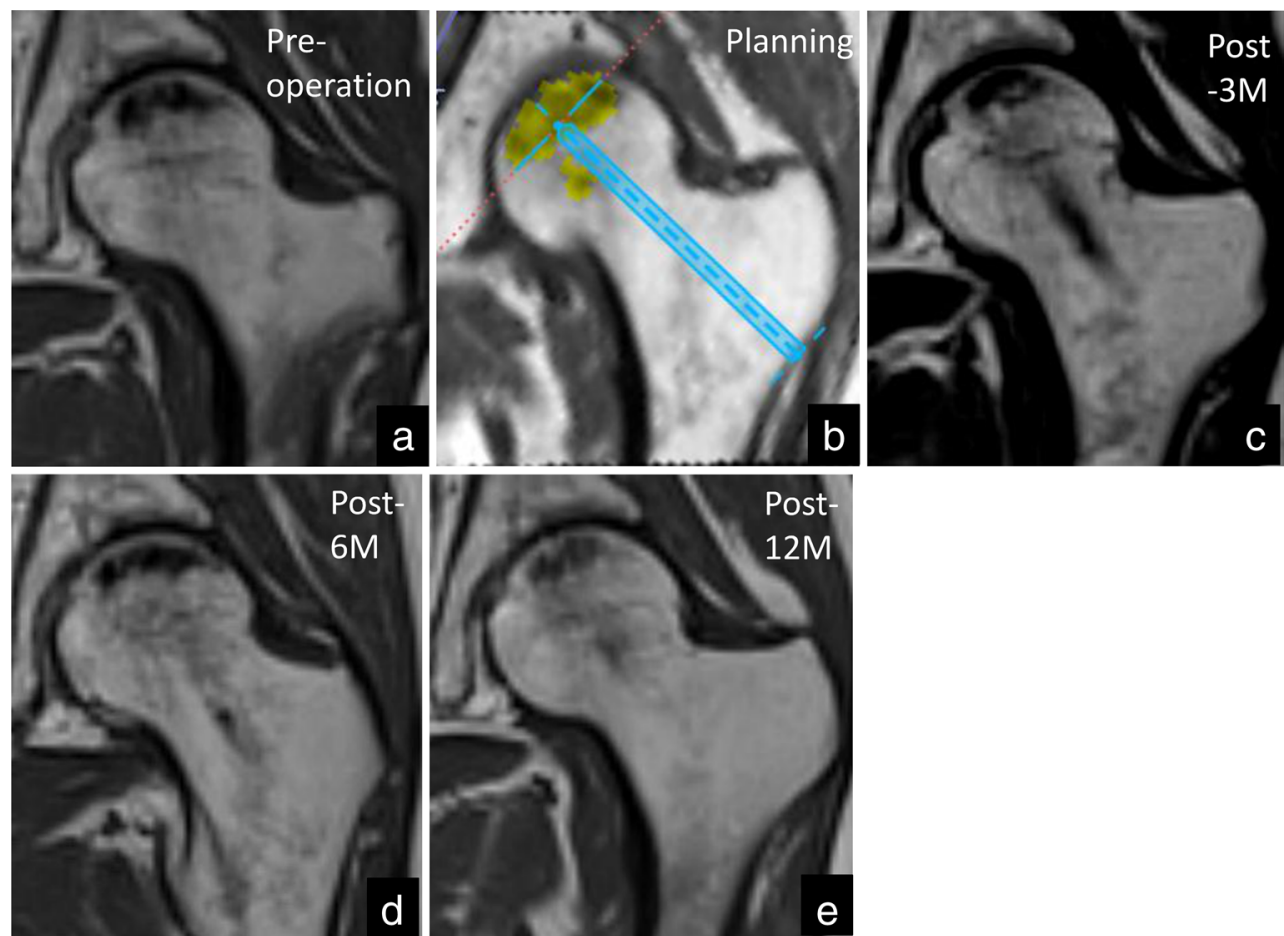

Fig. 2 Representative magnetic resonance images of the central coronal plane in the femoral head. a Pre-operative coronal T1-weighted magnetic resonance imaging (MRI) showing type $\mathrm{C} 2$ osteonecrosis of the femoral head (ONFH) occupying more than the medial two-thirds of the weightbearing portion and extending laterally to the acetabular edge. b MRI of the pre-operative plan described in Fig. 1. c MRI of the same femoral neck and intertrochanteric region at three months post-operatively, showing low signal intensity, indicating the drilling route made during the surgical procedure. $\mathbf{d}$ MRI at six months post-operatively showing the first change of signal intensity in the drilling route. The signal intensity of bone at the drilling site of the femoral neck is normalizing. e MRI at 12 months post-operatively showing almost normal signal intensity in the femoral neck. The area and size of ONFH remains unchanged from type 2. Abbreviations: M, months 


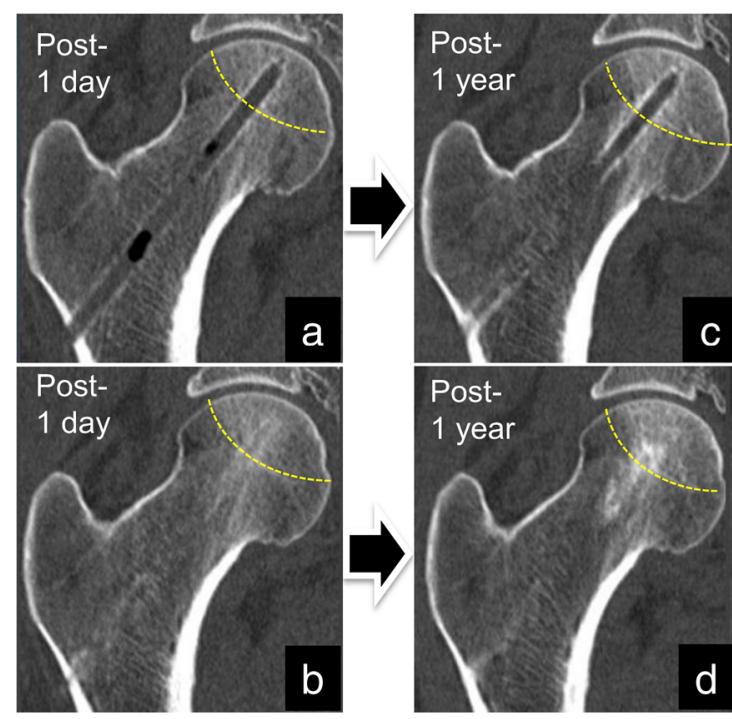

Fig. 3 Two representative computed tomography (CT) images with the binary format data of the central implanted area. The yellow dashed line shows the border of the osteonecrotic area of the femoral head. These images are for the same patient shown in Fig. 2a. Coronal CT images show the bone defect at the implanted region at day one post-operatively $(\mathbf{a}, \mathbf{b})$, and apparent bone regeneration is observed one year post-operatively (c, d). The normal contour of the femoral head is maintained

secondarily because of the lack of a technique to locally deliver the growth factor [17]. The angiogenic and osteogenic actions of rhFGF-2 have been the subject of numerous reports for bone and joint disease. In particular, when used with gelatin hydrogel as a slow-release carrier, treatment with rhFGF2 in animal experiments has been shown to increase bone mass in deficit areas $[9,11,12]$. Release of rhFGF-2 from the hydrogel at the site of implantation was measurable for more than 2 weeks, a period that correlates strongly with the patterns of in vivo rhFGF-2 release and hydrogel degradation [18]. One human application that merits attention is a report on single injections of rhFGF-2-impregnated hydrogel at doses of 200,400 , and $800 \mu \mathrm{g}$ in cut bone surfaces of the lower leg, which produced rapid synostosis in a dosedependent manner [19, 20].

The most important limitation of the present study is the lack of a control group using the gelatin hydrogel alone. In terms of minimally invasive therapies, the simplest technique is core decompression, which has been used for a long time. This method creates a burr hole in the femoral head with the anticipation that self-healing will occur; however, it cannot ultimately prevent femoral head collapse. High collapse rates have been reported in up to $72.7 \%$ of femoral heads after use of core decompression alone [5]. Although it is performed relatively frequently in Europe and the United States, core depression is rarely used in Japan unless bone biopsy is necessary. Therefore, it was considered that using a control group with gelatin hydrogel would present ethical problems in this clinical trial. Therefore, we simply provided an $800-\mu \mathrm{g}$ dose of rhFGF-2, as determined from preclinical experiments using

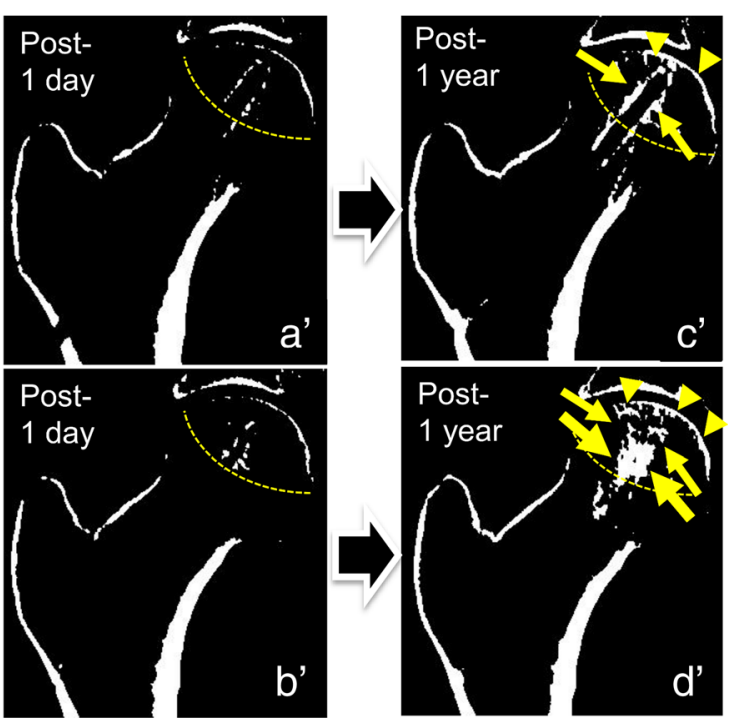

throughout. In the adjoining images for the binary format data, thin trabecular bone and subchondral bone can be seen in the implanted region at day 1 postoperatively $\left(a^{\prime}, b^{\prime}\right)$, while apparent bone regeneration is observed in the implanted region at one year post-operatively (yellow arrows, $\left.\mathrm{c}^{\prime}, \mathrm{d}^{\prime}\right)$. Clear, normal-contour, thick trabecular bone and thick subchondral bone (yellow arrowheads) can be observed

an animal model of ONFH, without a control group. Other limitations include the small sample size and short follow-up period; this was because, as a phase II study and the first clinical trial in humans, this study was performed mainly to evaluate safety within a strict budget.

In Europe and the United States, novel therapies have been developed recently for the management of ONFH, such as autologous bone marrow cell transplantation and embedding of metal implant rods. Autologous bone marrow cell transplantation may be reliable for the treatment of the precollapse stage of ONFH [4-6]. Implantation of cylindrical metal (porous tantalum) rods into the femoral head can prevent collapse by providing structural support in the necrotic area, which has been approved as an early-stage therapy [21, 22]. However, we believe that direct administration of rhFGF-2-impregnated gelatin hydrogel offers the important advantages of not only being a minimally invasive percutaneous technique that facilitates early return to society but also being able to promote bone regeneration in necrotic areas. Therefore, with further development, it could become a useful treatment for the precollapse stage of ONFH because it can be performed any time before femoral head collapse, regardless of the cause.

Acknowledgments We thank M. Doi and K. Enomoto for data management and T. Ikeda for clinical trial design and management.

\section{Compliance with ethical standards}

Funding This work was supported in part by grants from Translational Research Network Program by Ministry of Education, Culture, Sports, 
Science and Technology of Japan and The Uehara Memorial Foundation (to A.H.).

Conflict of interest The authors declare that they have no conflict of interest.

Open Access This article is distributed under the terms of the Creative Commons Attribution 4.0 International License (http:// creativecommons.org/licenses/by/4.0/), which permits unrestricted use, distribution, and reproduction in any medium, provided you give appropriate credit to the original author(s) and the source, provide a link to the Creative Commons license, and indicate if changes were made.

\section{References}

1. Hernigou P, Ooignard A, Nogier A, Manicom O (2004) Fate of very small asymptomatic stage-I osteonecrotic lesions of the hip. J Bone Joint Surg Am 86:2589-2593

2. Mont MA, Jones LC, Hngerford DS (2006) Nontraumatic osteonecrosis of the femoral head: Ten years later. J Bone Joint Surg Am 88:1117-1132, Review Erratum in: J Bone Joint Surg Am 88A:1602

3. Marker DR, Seylee TM, McGrath MS, Delanois RE, Ulrich SD, Mont MA (2008) Treatment of early stage osteonecrosis of the femoral head. J Bone Joint Surg Am 90:175-187

4. Hernigou P, Flouzat-Lachaniette $\mathrm{CH}$, Delambre J, Poignard A, Allain J, Chevallier N, Rouard H (2015) Osteonecrosis repair with bone marrow cell therapies: State of the clinical art. Bone 70:102109

5. Gangji V, De Maertelaer V, Hauzeur JP (2011) Autologous bone marrow cell implantation in the treatment of non-traumatic osteonecrosis of the femoral head: five year follow-up of a prospective controlled study. Bone 49:1005-1009

6. Houdek MT, Wyles CC, Martin JR, Sierra RJ (2014) Stem cell treatment for avascular necrosis of the femoral head: current perspectives. Stem Cells Clon 7:65-70

7. Lieberman JR, Conduah A, Urist MR (2004) Treatment of osteonecrosis of the femoral head with core decompression and human bone morphogenetic protein. Clin Orthop Relat Res 429: 139-145

8. Sun W, Li Z, Gao F, Shi Z, Zhang Q, Guo W (2014) Recombinant human bone morphogenetic protein- 2 in debridement and impacted bone graft for the treatment of femoral head osteonecrosis. PLoS One 9:e100424

9. Kuroda Y, Akiyama H, Kawanabe K, Tabata Y, Nakamura T (2010) Treatment of experimental osteonecrosis of the hip in adult rabbits with a single local injection of recombinant human FGF-2 microspheres. J Bone Miner Metab 28:608-616

10. Sugano N, Atsumi T, Ohzono K, Kubo T, Hotokebuchi T, Takaoka $\mathrm{K}$ (2002) The 2001 revised criteria for diagnosis, classification, and staging of idiopathic osteonecrosis of the femoral head. J Orthop Sci 7:601-605

11. Tabata Y, Ikada Y (1999) Vascularization into a porous sponge by sustained release of basic fibroblast growth factor. Biomaterials 20: 2169-2175

12. Tabata Y, Yamada K, Hong L, Miyamoto S, Hashimoto N, Ikada Y (1999) Skull bone regeneration in primates in response to basic fibroblast growth factor. J Neurosurg 91:851-856

13. Amstutz HC, Thomas BJ, Jinnah R, Kim W, Grogan T, Yale C (1984) Treatment of primary osteoarthritis of the hip. A comparison of total joint and surface replacement arthroplasty. J Bone Joint Surg Am 66:228-241

14. Harris WH (1969) Traumatic arthritis of the hip after dislocation and acetabular fractures: treatment by mold arthroplasty. An endresult study using a new method of result evaluation. J Bone Joint Surg Am 51-A:737-755

15. Cnaan A, Laird NM, Slasor P (1997) Using the general linear mixed model to analyse unbalanced repeated measures and longitudinal data. Stat Med 16:2349-2380

16. Poignard A, Lebouvier A, Cavet M, Rahmouni A, Flouzat Lachaniette CH, Bierling P, Rouard H, Hernigou P, Chevallier N (2014) New preclinical porcine model of femoral head osteonecrosis to test mesenchymal stromal cell efficiency in regenerative medicine. Int Orthop 38(9):1837-1844

17. Cheng TL, Murphy CM, Cantrill LC, Mikulec K, Carpenter C, Schindeler A, Little DG (2014) Local delivery of recombinant human bone morphogenetic proteins and bisphosphonate via sucrose acetate isobutyrate can prevent femoral head collapse in LeggCalve-Perthes disease: a pilot study in pigs. Int Orthop 38(7): $1527-1533$

18. Yamamoto M, Ikeda Y, Tabata Y (2001) Controlled release of growth factors based on biodegradation of gelatin hydrogel. J Biomater Sci Polym Ed 12:77-88

19. Kawaguchi H, Jingushi S, Izumi M, Fukunaga M, Matsushita T, Nakamura T, Mizuno K, Nakamura T, Nakamura K (2007) Local application of recombinant human fibroblast growth factor-2 on bone repair: a dose-escalation prospective trial on patients with osteotomy. J Orthop Res 25:480-487

20. Kawaguchi H, Oka H, Jingushi S, Izumi T, Fukunaga M, Sato K, Matsushita T, Nakamura K, TESK Group (2010) A local application of recombinant human fibroblast growth factor 2 for tibial shaft fractures: a randomized, placebo-controlled trial. J Bone Miner Res 25:2735-2743

21. Tsao AK, Roberson JR, Christie MJ, Dore DD, Heck DA, Robertson DD, Poggie RA (2005) Biomechanical and clinical evaluations of a porous tantalum implant for the treatment of early-stage osteonecrosis. J Bone Joint Surg Am 87(Suppl 2):22-27

22. Malizos KN, Papasoulis E, Dailiana ZH, Papatheodorou LK, Varitimidis SE (2012) Early results of a novel technique using multiple small tantalum pegs for the treatment of osteonecrosis of the femoral head: a case series involving 26 hips. J Bone Joint Surg (Br) 94:173-178 